\title{
A Novel Automated Approach for Offline Signature Verification Based on Shape Matrix
}

\author{
Sumbal Iqbal Ahmed \\ Abasyn University \\ Peshawar Pakistan
}

\author{
Zuhaib Ahmad \\ Abasyn University \\ Peshawar Pakistan
}

\author{
Rashid Jalal Qureshi \\ Emirates Aviation University \\ Dubai ,UAE
}

Imran Khan

Abasyn University

Peshawar, Pakistan

\author{
Abdus Salam \\ Abasyn University \\ Peshawar Pakistan
}

\begin{abstract}
The handwritten signature has been the most natural and long lasting authentication scheme in which a person draw some pattern of lines or writes his name in a different style. The signature recognition and verification are a behavioural biometric and is very challenging due to the variation that can occur in person's signature because of age, illness, and emotional state of the person. As far as the representation of the signature is concerned a classical technique of thinning or skeleton is mostly used. In this paper, we proposed a new methodology for signature verification that uses structural information and original strokes instead of skeleton or thinned version to analyse the signature and verify. The approach is based on sketching a fixed size grid over the signatures and getting 2-Dimensional unique templates which are then compared and matched to verify a query signature as genuine or forged. To compute the similarity score between two signature's grids, we follow template matching rule and the Signature grid's cell are mapped and matched with respect to position. The proposed framework is fast and highly accurate with reduce false acceptance rate and false
\end{abstract}

Keywords: Authentication, Biometric identification, Feature extraction, Signature verification, Shape matrix.

\section{INTRODUCTION}

There are many authentication schemes like fingerprints, voice recognition, iris scanning, retina scan, face recognition etc., however, a handwritten signature has already become an accepted proof of identity of the person in our daily life, especially in financial sectors, where a transaction taken on his or her behalf are being authorized via signatures.

A handwritten signature is the scripted name or legal mark of a person's identity, executed by hand. The signature of a person cannot be stolen, however, its forgery is possible after a good practice. To make a duplicate or false copy of a person's signature the forger tries to produce the signature as closest to the original by carefully learning the basic style of writing and shape characteristics of a signature. Also, the signature recognition and verification are a behavioral biometric and is very challenging due to the variation that can occur in person's signature because of age, illness, and emotional state of the person. So, it is needed to design a system that verifies the signature of a human automatically. It can be operated either as "off-line" signature verification or "on-line" signature verification. In an off-line signature verification, the user write a signature on paper which is then digitize by an optical scanner or a camera, and the biometric system identifies the signature by analyzing its shape. The static information derived in an offline signature verification system may be global, structural, geometric or statistical. On contrary, in an On-line signature verification, data records the motion of the stylus when the signature is produced, and includes location, and possibly velocity, acceleration and pen pressure, as functions of time. Processing of offline signature is complicated because no stable dynamic features are available and segmenting the signature strokes is very difficult because of variation in writing styles of each person and the variation that can occur in person's signature. Therefore, main challenging problem in design of an offline signature verification system is the phase of extracting features that distinguish between forged and genuine signatures. In this paper, a novel method based on shape matrix is proposed. The proposed technique of signature verification is simple and quick with an excellent recognition rates.

This paper is organized as follow: Section 2 is about the literature review and survey of previously presented techniques for signature verification. Section 3, introduces the proposed model and pre-processing used. Section 4, explain the use of shape matrix in the domain of signature. Section 5, presents the results of our method and its comparisons with the existing similar approaches. Section 6, briefly conclude this research work and point out some possible future work.

\section{RELATED WORKS}

Many research works on signature verification have been reported. Researchers have applied many technologies, such as neural networks [1] [2], Hidden Markov Model [3] and Support Vector machine [4] and pixel based [5] processing to the problem of signature verification and they are continually introducing new ideas, concepts, and algorithms. 
As far as the representation of the signature is concerned a classical technique of thinning or skeletonization is used mostly. It consists of representing the original strokes of the signature by a thinnest representation but preserving the topology or basic structure. After thinning a set of idealized lines is obtained which is called the skeleton or medial axis.

In [6] the authors have used a similar approach of obtaining the skeleton of a signature by using thinning technique. Each pixel that belongs to the signature is then studied and the extraction of endpoints from the signature geometry is done. These endpoints were then joined to draw a closed shaped polygon. The structural features of the polygon like area, perimeter, circularity measure, rectangularity measure, and minimum enclosing rectangle were taken into consideration. A verification function was built by the combination of these features and then its evaluation was done by Euclidean distance matrix.

On a similar note, [7] used signature's skeleton and produced a Delaunay triangulation with selected end points and intersection points. The technique is based on matching the triangles based on relative areas of the triangles and their mutual angles with neighboring triangles.

Kumar et al. [8] presented the technique of Off-line Signature Verification Based on Fusion of Grid and Global Features Using Neural Networks. The skeletonized image is divided into 120 rectangular segments (15x8), and for each segment, the area (the sum of foreground pixels) is calculated. The resulting 96 values form the grid feature vector. Some common global features such as Aspect Ratio, Signature height, Image area, pure width and height were used. The standard back propagation neural network classifier for verification is used. Multilayer feed forward artificial neural network for verification of off-line digitized signatures is used. The proposed NN consists of 30 input variables which are extracted from signature features, and it is designed to verify one signature at a time. Back propagation algorithm is used for training.

All the known thinning techniques generally produce rough branches at the crossing points and junction of lines, which are called bushes. It is fact that two signatures of the same person do have some variations. These small changes in lines can disturb the skeleton a lot and the features based on skeleton points will not be same. This leads to low recognition rate. A technique for Off-line Verification of signatures using a collection of simple shape based geometric features was presents in [9]. The geometric features that are used are a Centre of gravity, Area, Eccentricity, Kurtosis, and Skewness. ANN (artificial neural network) was used to confirm and classify the signatures. In [10] another method for the classification and verification using feature point is presented. The scheme is based on selecting 60 feature points from the geometric center of the signature and compares them with the already trained features points.

In [11] the topological and texture features are extracted from the actual signature set. The system is trained by using these features. The mean feature values of all the actual signature features are calculated. This mean features acts as the model for verification against a test signature. Euclidian distance between template signature features and claimed signature features serves as a measure of similarity between the two. In pre-processing, the binary signature image is cropped to keep only the signature as the content, without noise removal, the features that are based on pixels can results in wrong selections. The system performance deteriorates in case of skilled forgeries too.

Roy et al [12] presented a method of handwritten signature verification using grid based approach and pixel oriented approach. Intersecting points and centroids of two equal half of the signature is being calculated and then those centroids are connected with a straight line and the angles of these intersecting points with respect to the centroids connecting lines are calculated. However, the framework does not produced impressive results, works average in simple forgery to produce a low FAR but skilled forgery case produces $20 \%$ FAR.

\section{PROPOSED MODEL AND IMPLEMENTATION}

The steps involved in the proposed strategy for off-line signature verification based on shape matrix are shown in the block diagram (e.g. Figure 1). The approach is based on sketching a fixed size grid over the signatures and getting a 2-Dimensional simplified templates which are then compared and matched to verify a query signature as genuine or forged.

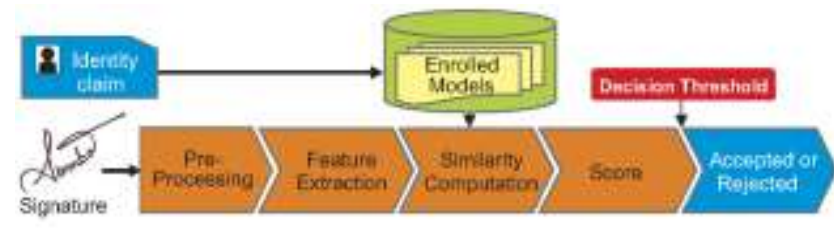

Figure 1 . Proposed Methodologies

\subsection{Pre-processing}

\subsubsection{Converting colour image into binary}

In contrast to colour or grey image, handling the binary image is easier and simpler because the image will be in 2-bit representation. Therefore, the colour image is first converted into grey image by setting the intensities of red, green and blue in $30 \%, 60 \%$ and $11 \%$ respectively. Then, the Otsu's method [13] was used, which chooses the threshold to minimize the intra-class variance of the black and white pixels. The image obtained after this will be a 2-Dimensional image in which black colour is represented by 0 value (the signature strokes) and white colour is represented by 255 value - the background. However, the inverted image is needed for further processing therefore the image is inverted that is the black pixels are turned into white pixels and vice versa (e.g. Figure 2).

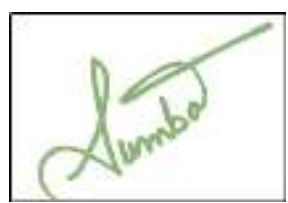

(a)

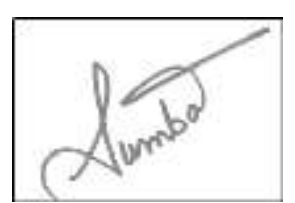

(b) 


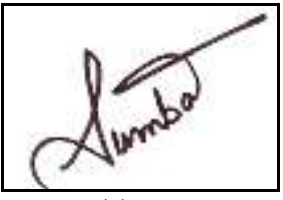

(c)

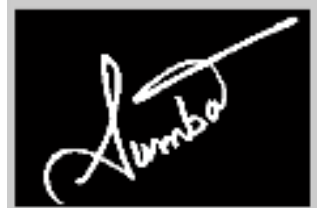

(d)
Figure 2. a) Scanned image b) Gray scale image c) Binary image d) Inverted image

\subsubsection{The Slant Removal and Region of Interest (ROI) in the Signature Image:}

It is a common and natural that different people sign at different angle and most of the time it is not straight or horizontal to $\mathrm{x}$-axis. But the signature is usually comprised of more than one connected components. We need the signature parts fused together such that we can treat it as one blob or connected area, find its orientation and do the rotation such that it should parallel to horizontal $\mathrm{X}$-axis.

For this purposed, we have used the concept of morphological closing. The orientation angle is found by fitting an ellipse, the major axis of the ellipse shows orientation to $\mathrm{X}$-axis. Once the angle is calculated the image is rotated clock wise or counter clockwise such that it is laying at an angle zero degree or parallel to $\mathrm{X}$-axis as shown in Figure 3.

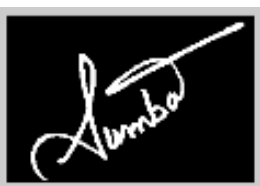

(a)

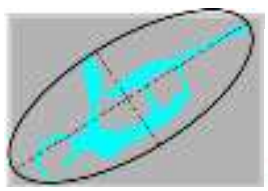

(c) (b)

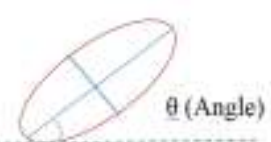

(d)

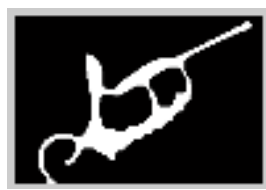

Figure 3. Slant removal, a) signature b) after morphological closing c) ellipse fitting d) angle computed: 35.1679 o

A signature may have more than one disconnected regions or parts and hence we can have more than one minimum bounding box enclosing each region (e.g. Figure $4 a$ ). We need one bounding box that encapsulates the entire signature. To calculate the minimum enclosing rectangle (MER), all the rectangles corners were compared and maximum and minimum $\mathrm{x}$ and $\mathrm{y}$ coordinates were selected. These values will correspond to top left and bottom right of the minimum bounding box (e.g. Figure 4b).

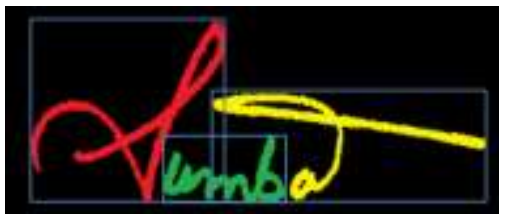

a)

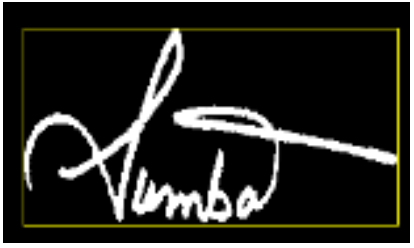

b)

Figure 4. Region of Interest - ROI, a) MER enclosing each region b) MER enclosing signature

\subsubsection{Image Normalization (resizing)}

The size of the same person signature may vary. So normalization must be done to scale all the signatures images to a fixed size and minimize the problems that may arise due to difference in size of the signature at the time of comparison. We have used the reference size $128 * 256$ in the proposed approach.

\section{SIGNATURE SHAPE MATRIX}

\subsection{Morphological operators instead of thinning} In our approach, we have not used thinning technique as important information is lost and it produce bushes effect on intersections (e.g. Figure 5b). The great amount of information can be saved by using bridge and remove operator, since the signature boundary is kept saved. Removal of pixels from the boundary of signature can be done but objects are not break apart or separated, (e.g. Figure 5d) shows remove operator's effect.

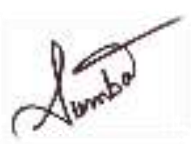

(a)

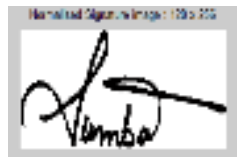

(c)

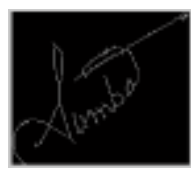

(b)

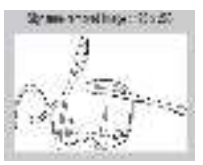

(d)
Figure 5. a) Original signature b) Signature image after thinning c) Normalized Signature d) Signature image after applying morphological operation

\subsubsection{Signature’s Shape Matrix}

The proposed signature representation involve division of signature into square grid of size $\mathrm{N} \times \mathrm{N}$. In this case, we have used $\mathrm{N}=10$. Hence, the signature is divided into a matrix having 10 rows and 10 columns using equal horizontal density method. For each block/cell in the matrix, the signature normalized area is computed. The normalized area is calculated by taking sum of pixels in each box and dividing it by total number of pixels in the signature. The block/cell having area greater than threshold are set to one (1) while the other are set to zero (0). Thus we obtained, for each signature, a binary matrix of $10 \times 10$ representing a compact signature code, (e.g. Figure. 6) shows two such signatures and their binary matrix codes. 


\subsubsection{Similarity Score - Signature's Grid \\ Matching}

To compute the similarity between two signature's grids, we follow template matching rule and the Signature grid's cell are mapped and matched with respect to position. For this purpose, simultaneously, the two grids i.e., the Test

Signature's grid and the kth signature grid's cells are scanned and compared for a possible match. If the Test signature grid cell's data is equal to the kth signature grid cell data the score is incremented as a step function. The Match Function is

$\operatorname{Match}(i, j)= \begin{cases}1, & \text { Test }(i, j) \text { equal } K(i, j) \\ 0, & \text { otherwise }\end{cases}$

A variable Match $(i, j)$ has a value of one (1) if the data is equal i.e., both grid's cell has a 0 and both grid's cell has a 1 . Score is computed as an aggregation function based on the number of times the data in the two grids matches.

$$
\text { Score }_{t, k}=\sum_{i=1}^{n} \sum_{i=1}^{m} \operatorname{Match}(i, j)
$$

Where $\mathrm{n}$ represents the number of rows and $\mathrm{m}$ represents number of columns in the grid. The grid similarity computed in percentage is a normalized score between the two grids showing the percentage match and closeness of the two signature. The Grid similarity is given by

$$
\text { Grid }_{\text {Similarity t,k }}=\frac{\text { Score }_{t, k}}{n * m} \times 100
$$

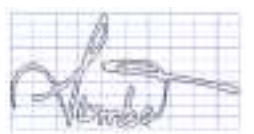

a) Signature

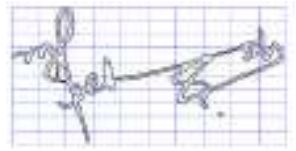

c) Signature

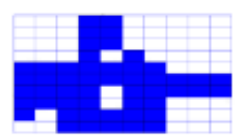

b) Shape matrix for signature

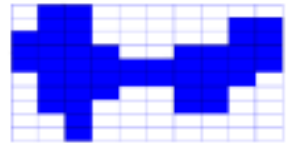

d) Shape matrix for signature
Figure 6. Signature with their binary shape matrix - the template, Grid Matching Score is: $53 \%$ Match

We have taken ten (10) sample signatures from each individual and it was encouraging to notice that the scores for the same person were quite higher than the score of similarity between two different person's signatures, it shows the power of discrimination of the proposed approach (e.g. Figure 7).

\section{RESULTS AND DISCUSSIONS}

Due to unavailability of the standard database, we gathered our own database. This database consists of 1000 signatures that are categorized into 50 classes with each class containing 20 signatures. These are the signatures belonging to 50 subjects and for each person there are 10 genuine signatures and 10 forgery signatures.

Since generally one person can have minor variations in his or her signatures in different trials, each individual was asked to provide 10 samples in multiple sessions over up to 2 weeks period. Ten of the people were requested to give a set of simple forgeries, and the other ten experts were asked to give a set of skilled forgeries to the signature of the 50 subjects.

If the grid matching score of the query signature image with respect to models signature image is below the threshold range, the query signature is detected as forged otherwise it is detected as genuine one. There are three different percentages that have been used to measure the performance. These are False Acceptance Rate (FAR), False Rejection Rate (FRR), and Accuracy. FAR is the percentage of forgeries that are incorrectly classified.

$F A R=\frac{\text { number of forgeries accepted }}{\text { number of forgeries tested }} \times 100$

FRR is the percentage of original signatures that are incorrectly classified.

$$
F A R=\frac{\text { number of original rejected }}{\text { number of original tested }} \times 100
$$

Accuracy is the percentage of signatures which are exactly classified. The Score of similarity in Table. 1 for same person matching is always above $90 \%$ an indication that the proposed method is capable of ignoring slight variation that a person usually have among his/her signature.

\begin{tabular}{|l|l|l|}
\hline Signature Image & Signature Shape Matrix & $\begin{array}{l}\text { Score } \\
\text { with }\end{array}$ \\
S1-1.jpg
\end{tabular}




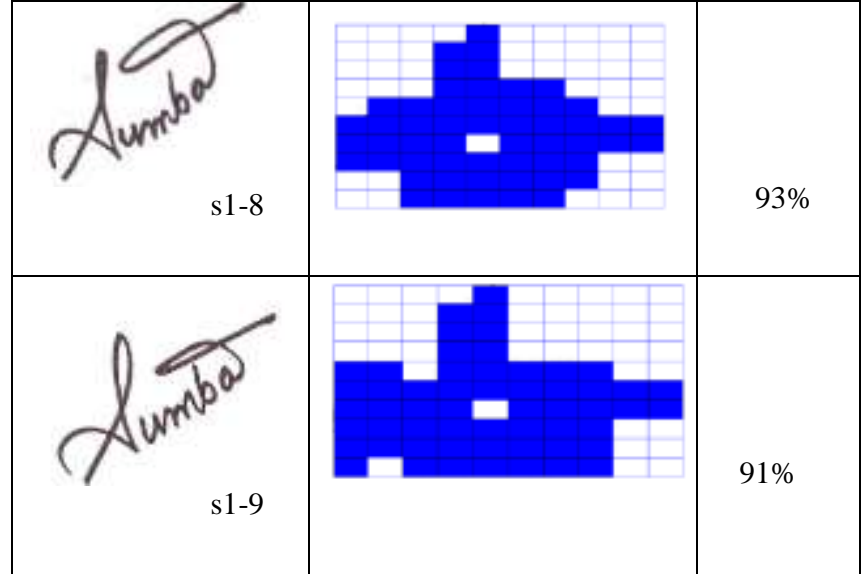

Figure 7. Score of similarity between signatures of same person

Table 1 . Similarity score for the same person's 10 signature

\begin{tabular}{|c|c|c|c|c|c|c|c|c|c|c|}
\hline & 31.1 & 81.2 & 513 & 51.4 & a1.5 & $51-6$ & 81.7 & 81.8 & 81.9 & $11-10$ \\
\hline st-t & 100 & 0 & 0 & 0 & 0 & 0 & 0 & 0 & 0 & 0 \\
\hline $91-2$ & 99 & 100 & 0 & 0 & 0 & 0 & 0 & 0 & 0 & 0 \\
\hline $81-3$ & 98 & 93 & 100 & 0 & 0 & 0 & 0 & 0 & 0 & 0 \\
\hline $21-4$ & 95 & 94 & 90 & 100 & 0 & 0 & 0 & 0 & 0 & 0 \\
\hline $11-5$ & 90 & 91 & 90 & 92 & 100 & 0 & 0 & 0 & 0 & 0 \\
\hline $31-6$ & 92 & 97 & 94 & 91 & 96 & 100 & 0 & 0 & 0 & 0 \\
\hline 51.7 & 92 & 94 & 91 & 92 & 97 & 97 & 100 & 0 & 0 & 0 \\
\hline st-8 & 94 & 97 & 94 & 91 & 96 & 98 & 97 & 100 & 0 & 0 \\
\hline st. 9 & 94 & 97 & क6 & 91 & 96 & 96 & 97 & 92 & 100 & 0 \\
\hline $51-10$ & 94 & 91 & 98 & 91 & 94 & 92 & 95 & 90 & 92 & 100 \\
\hline
\end{tabular}

In contrast, the forgeries scores are in the range of $50 \%$ to $60 \%$ and can be easily detected by the proposed system (see Table. 2). The Score of similarity for inter-personal matching is always below $50 \%$ and in the range of 30 to 40 (Table. 3) shows the power of discrimination of the proposed method.
Table 2. The score of similarity for the same person's 10 forgeries

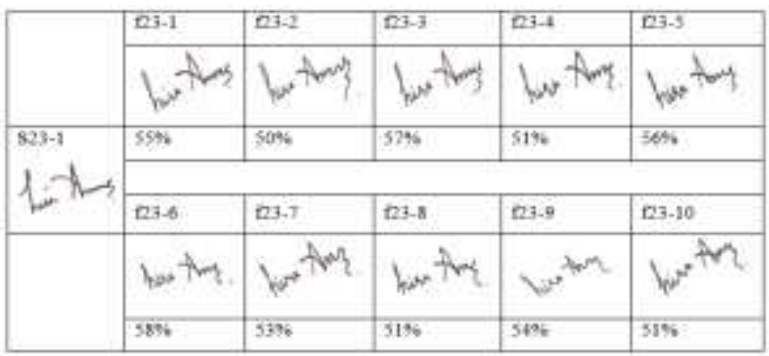

Table 3. The score of similarity for the inter-personal

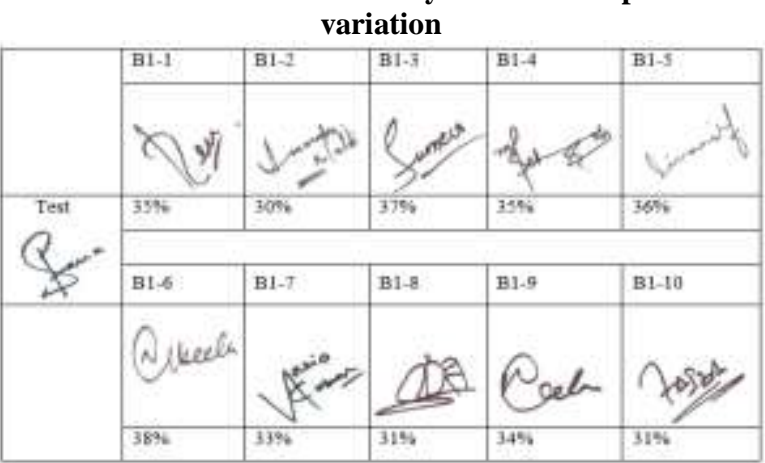

We have compared our algorithms with two methods one is the 60 feature points Scheme [10] and other is the end point polygon [6].

Our proposed algorithms that were based on structural features got better results than geometric features and end point polygon. The suggested algorithms have successfully rejected skilled forgeries with a very satisfying and good rate and have rejected the simple forgeries very perfectly as shown in Table. 4 and Table. 5. The values of FAR and FRR of our method is better than other schemes.

Table 4. Comparative Analysis of FAR

\begin{tabular}{|l|l|l|l|}
\hline $\begin{array}{l}\text { Forgery } \\
\text { Type }\end{array}$ & $\begin{array}{l}60 \\
\text { feature } \\
\text { points } \\
\text { scheme } \\
{[10]}\end{array}$ & $\begin{array}{l}\text { End } \\
\text { points } \\
\text { polygon } \\
{[6]}\end{array}$ & $\begin{array}{l}\text { Proposed } \\
\text { Method } \\
\text { (Shape } \\
\text { Matrix })\end{array}$ \\
\hline Simple & 0.98 & 0.73 & 0.65 \\
\hline Skilled & 2.08 & 1.57 & 1.43 \\
\hline
\end{tabular}


Table 5. Comparative Analysis of FRR

\begin{tabular}{|l|l|}
\hline \multicolumn{2}{|c|}{ False Rejection Rate (FRR) } \\
\hline 60 feature points scheme [10] & 20.83 \\
\hline End points polygon [6] & 16.35 \\
\hline Proposed Method (Shape Matrix) & 4.15 \\
\hline
\end{tabular}

\section{CONCLUSION}

Processing of offline signature is complicated because no stable dynamic features are available and segmenting the signature strokes is very difficult because of variation in writing styles of each person and the variation that can occur in person's signature.

The objective of the research was to develop an algorithm which should be relatively fast. We were able to design an offline signature verification system which is highly accurate with reduce false acceptance rate and false rejection rate. The proposed method opens a new area of research by using the original signature stroke and not its thin version or skeleton.

\section{REFRENCES}

[1] Malekian, V., Aghaei, A., Rezaeian, M. \& Alian, M.,2013,Rapid Off-line Signature Verification Based on Signature Envelope and Adaptive Density Partitioning. In IEEE conference on Pattern Recognition and Analysis (PRIA)., pp. $1-6$.

[2] Abdala, M. A., \& Yousif, N.M., 2009, Offline Signature Recognition and Verification Based on Artificial Neural Network. In Eng \& Tech. Journal., Vol. 27. No. 7.

[3] Edson, J., Justino, R.,Bortolozzi, F., \& Sabourin, R., 2005,A comparison of SVM and HMM classifiers in the offline signature verification. In Pattern Recognition Letters journal. Vol.26 Issue 9. pp.1377-1385.

[4] Ferrer, M. A., Alonso, B., \& Travieso, C.M., 2005,Offline Geometric Parameters for Automatic Signature Verification Using Fixed-Point Arithmetic., In IEEE transactions on pattern analysis and machine Intelligence., Vol. 27. No. 6.

[5] Barua, A., Hoque, M.M., Nurul, A.F.M., \& Habib, Md.A., 2015, Pixel Based Off-line Signature Verification System. In American Journal of Engineering Research (AJER)., e-ISSN : 2320-0847., pISSN : 2320-0936., Vol. 04. Issue-01.pp.187-192.
[6] Zafar, S., Qureshi, R.J., 2009 , Off-line signature verification using structural Features. In FIT '09, 7th International Conference on Frontiers of Information Technology, Abbottabad, Pakistan.

[7] Jan, Z., Muhammad, H., Rafiq,M., \& Zada, N., 2015,An automated System for offline signature verification and Identification using Delaunay Triangulation. In Advances in Intelligent Systems and Computing Journal., pp. 653-663.

[8] Kumar,S., Raja, K.B., Chhotaray, R.K., \& Pattanaik, S.,2010,Off-line Signature Verification Based on Fusion of Grid and Global Features Using Neural Network. In International Journal of Engineering Science and Technology.,Vol. 2(12)., pp.7035-7044.

[9] Karouni, A., Daya, B., \& Bhalak ,S.,2011,Offline signature recognition using neural networks approach. In Procedia Computer Science world conference on Information technology.,vol. 3.pp. 155-161.

[10] Jena, D., Majhi, Panigrahy,S.K., \& Jena, S.K., 2008, Improved offline signature verification Scheme using feature point extraction method. In Cognitive Informatics 7th IEEE International Conference on Cognitive Informatics (ICCI-08). pp.475-480.

[11] Jana, R., Mandal, S., \& Chaya, K., 2015, Offline Signature Verification for Authentication. In International Journal of Computer Applications., Vol. 126 .No. 6. Pp.20-23.

[12] Roy, S., Maheshkar, S., 2014, Offline Signature Verification using Grid based and Centroid based Approach. In International Journal of Computer Applications. Vol. 86. No 8.

[13] N. Otsu. A Threshold Selection Method from Gray-Level Histograms. 